\title{
Imaging spectroscopy of Ag plasmas produced by infrared nanosecond laser ablation
}

\author{
J. J. Camacho ${ }^{1 *}$, M. Oujja ${ }^{2}$, M. Sanz ${ }^{2}$, A. Martínez-Hernández ${ }^{2}$, I. Lopez-Quintas ${ }^{2 \ddagger}$, \\ R. de Nalda ${ }^{2}$, M. Castillejo ${ }^{2}$ \\ ${ }^{1}$ Departamento de Química-Física Aplicada. Facultad de Ciencias, Universidad \\ Autónoma de Madrid, Cantoblanco, 28049 Madrid, Spain \\ ${ }^{2}$ Instituto de Química Física Rocasolano, CSIC, c/ Serrano 119, 28006, Madrid, Spain \\ ${ }^{*}$ Present address: Elettra Sincrotrone Trieste, SS 14- Km 163.5, 34149 Basovizza, \\ Trieste, Italy
}

\begin{abstract}
Spatially and temporally resolved optical emission spectroscopy has been used to study plasmas formed by $1064 \mathrm{~nm}$ pulsed laser ablation of silver targets in vacuum. Ag and $\mathrm{Ag}^{+}$species were mapped as a function of both locations from the surface of the target and time from the laser pulse. Plasma parameters such as temperature and electron density were measured from the spatiotemporal analysis. We examined the properties of $\mathrm{Ag}$ resonance and non-resonance emission lines and $\mathrm{Ag}^{+}$ions. The results show a faster decay of the continuum emission and $\mathrm{Ag}^{+}$species than in the case of neutral $\mathrm{Ag}$ atoms. Higher velocities and a narrower temporal distribution of $\mathrm{Ag}^{+}$are observed in vacuum when compared to Ag atoms. Electron temperature was assessed from the comparison of Ag atomic/ionic Saha-local thermodynamic equilibrium spectrum with experimental ones at various delay times from the laser pulse incidence. An initial electron temperature of $2 \mathrm{eV}$ and electron density of $2 \times 10^{18} \mathrm{~cm}^{-3}$ were measured from the spatially-integrated spectra.
\end{abstract}

\footnotetext{
Keywords: Laser ablation; Laser spectroscopy; Atomic spectroscopy; Silver, Ag

*Corresponding author. Tel.: +34 91 3974960; fax: +34 913974512

E-mail address: j.j.camacho@uam.es
} 


\section{Introduction}

Pulsed laser ablation (PLA) is the process of removing material from a solid or occasionally liquid surface by irradiating it with a high-power pulsed laser. This technique for depositing and processing materials is an important area of modern experimental and theoretical scientific research and development, with challenging applications in the fields of pulsed laser deposition, ${ }^{1}$ laser-induced breakdown spectroscopy, ${ }^{2,3}$ surface modification, ${ }^{4}$ nanofabrication and nanostructuring, ${ }^{5}$ laser propulsion, ${ }^{6}$ medicine, ${ }^{7,8}$ environmental analysis ${ }^{9}$ and harmonic generation. ${ }^{10,11}$ Understanding the fundamental mechanisms and properties of laser-material interactions, as well as deposition and processing conditions, laser parameters and material properties, is essential for improving fundamental knowledge and applications. In spite of PLA applications, however, some aspects of the underlying physics remain incompletely understood, and the dynamics of the laser-produced plasma (LPP) remain a particular focus of study. Analysis of LPPs can be a very puzzling task considering the involved complex processes, its transient nature and the large variations in plasma properties with space and time. Optical emission spectroscopy (OES) is a useful technique to study the dynamics of species formed in the plasma induced in the ablation of materials.

Silver is a soft, very ductile, malleable, lustrous transition metal with the highest electrical and thermal conductivity and reflectivity of all metals. It is used as a precious metal, in solar panels, jewelry, water filtration, food additive/coloring, in electrical contacts and conductors, in superior mirror production, window coating, in catalysis of chemical reactions, among many other applications.

There is a moderate body of work diagnosing space and time resolved optical emission from silver LPP plumes ${ }^{12-16}$ for a range of experimental conditions and laser intensities. Previously, Issac et al. ${ }^{12}$ studied the dynamics of laser produced Ag plasma 
as a function of $\mathrm{Ar}$ ambient pressure. They observed an anomalous double peak intensity profile in the temporal optical time-of-flight profile of $\mathrm{Ag}$ atoms at low pressures. Margarone et al. ${ }^{13}$ studied the Ag plasma in a high-vacuum $\left(10^{-7} \mathrm{mbar}\right)$ using a ring ion collector and an electrostatic ion energy analyzer. In the same paper, the authors estimated an electron plasma temperature of about $5 \mathrm{eV}$ for the plasma corona region and $280 \mathrm{eV}$ for the plasma core from the time-integrated spectra obtained by using conventional OES. In addition, they also measured threshold fluence of silver. Shuaibov et al. ${ }^{14}$ studied time-averaged energy distributions and recombination times of Ag atoms. Musadiq et al. ${ }^{15}$ measured electron densities and temperatures from the timeintegrated spectra at different laser energies. Esposito et al. ${ }^{16}$ studied the $\mathrm{Ag}^{+}$dynamics of the pulsed laser ablation of $\mathrm{Ag}$ by using Langmuir probe and quadrupole mass spectrometry. They measured the kinetic energies and angular distributions of the ablated ions for different laser fluences.

In this work, we investigated the spatial and temporal dynamics of neutrals and singly ionized ions in a nanosecond laser (Nd:YAG, $1064 \mathrm{~nm}, 7 \mathrm{~ns}$, and an irradiance of $0.7 \mathrm{GW} \times \mathrm{cm}^{-2}$ ) induced silver plasma in vacuum using both OES and spectrally resolved two-dimensional (2D) imaging. Imaging OES studies confirm intense neutral line emission over ion emission. Analysis of the image spectra provides useful plasma parameters such as formed atomic/ionic species, average expansion velocities of some plasma species, electron density, and electron temperature. The results indicate a more rapid decay of the continuum emission and $\mathrm{Ag}^{+}$species than in the case of neutral $\mathrm{Ag}$ atoms. Moreover, higher rates and a narrower temporal distributions of $\mathrm{Ag}^{+}$ions are detected in vacuum when compared with $\mathrm{Ag}$ atoms. The rate of $\mathrm{Ag}$ atoms at earlier times, especially below $80 \mathrm{~ns}$, where the intense continuum is dominant, is nearly zero. Electron temperature was estimated by comparing $\mathrm{Ag}$ atomic/ionic Saha-local thermodynamic equilibrium (LTE) spectra with experimental ones at various delay 
times from the laser pulse incidence. This will supply much physical insight into evolution dynamics of neutrals and ionized species in a laser-produced silver plasma as it expands into vacuum.

\section{Experimental details}

The experimental setup used in this study is presented in Fig. 1. The $1064 \mathrm{~nm}, 7$ ns full width at half-maximum (FWHM) pulses from a Q-switched Nd:YAG laser (Spectra Physics, Quanta Ray Indi-HG) was used to create a silver plasma in a stainless steel vacuum chamber. A base pressure of $\approx 2 \times 10^{-5}$ mbar was maintained in all the experiments. A 2 mm thick 99.99\% pure silver target (from Kurt J. Lesker Company) in the form of a disc was rotated and translated to avoid errors due to local heating and drilling. Laser beam energy was monitored using an optical power meter (Newport, Model 1917-R). The laser beam was focused onto the target surface at normal incidence using an $f=20 \mathrm{~cm}$ anti-reflection coated plano-convex lens. The spot radius on the target surface was $400 \mu \mathrm{m}$. At the target position, the laser supplied $25 \mathrm{~mJ}$ (irradiance 0.7 $\mathrm{GW} \times \mathrm{cm}^{-2}$, fluence $5 \mathrm{~J} \times \mathrm{cm}^{-2}$, photon flux $4 \times 10^{27}$ photon $\times \mathrm{cm}^{-2} \times \mathrm{s}^{-1}$, and electric field 0.5 $\left.\mathrm{MV} \times \mathrm{cm}^{-1}\right)$. The laser was usually operated at a repetition rate of $10 \mathrm{~Hz}$ and the shot-toshot variability of the energy was $\approx 5 \%$.

For performing time and space resolved OES, the plasma emission was collected and imaged onto the entrance slit of a $0.3 \mathrm{~m}$ spectrograph (Bentham, TMc300). The spectrograph was equipped with three gratings although in all the experiments presented here, we used a grating of 1200 grooves $/ \mathrm{mm}$ with a spectral resolution of $\approx 0.3 \mathrm{~nm}$. We have set the origin of axial distance, $z=0 \mathrm{~mm}$, as the target surface. The spectra emitted by Ag LPPs have been studied in the spectral region from about 290 to $560 \mathrm{~nm}$. A quartz Dove prism was inserted into the optical path for capturing two-dimensional (2D) spectral imaging and rotating the plasma image by $90^{\circ}$, projecting the direction of 
the plasma expansion (Z-axis) onto the entrance slit. The Dove prism is placed between two lenses with focal distances of $8 \mathrm{~cm}$ (L1) and $4 \mathrm{~cm}$ (L2) being the magnification of the final image $0.5 \times$ or 1 (image size) $: 2$ (real size). The output of the spectrograph was coupled to an intensified charge-coupled device (ICCD) camera (Andor Technology, DH501-25F-03, 1024×128 active pixels, $26 \mu \mathrm{m}^{2}$ pixel, intensifier diameter $25 \mathrm{~mm}$ ). Wavelength dispersion in the spectrograph was recorded horizontally across the ICCD chip, while the plasma-plume length along the $\mathrm{Z}$ coordinate was recorded vertically. The maximum plasma height that can be measured is $6.6 \mathrm{~mm}$. The spectra were recorded by the ICCD which operated in imaging mode. In order to enhance the signalto-noise ratio, data acquisition was performed by accumulating the signal over 125 successive laser shots. The ICCD detector is synchronized with the trigger of the laser pulse and gated through a digital delay/pulse generator (Stanford DG 535) connected to the Q-Switch output electrical signal of the laser. The exposure time of the image intensifier, typically referred to as the gate width time $t_{\mathrm{w}}$, was $100 \mathrm{~ns}$. The delay time $t_{\mathrm{d}}$ after the beginning of the laser pulse was monitored from 0 to $1500 \mathrm{~ns}$.

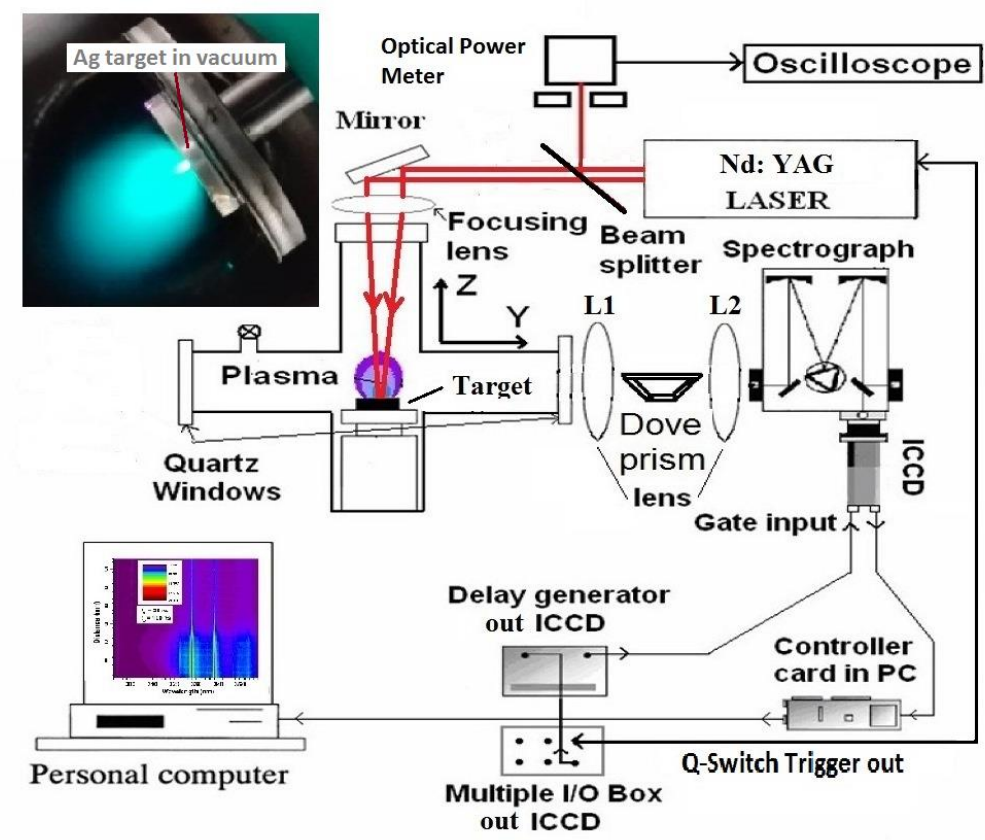

Fig. 1. Schematic diagram of the experimental setup of space and time gated ICCD for Nd: YAG pulsed laser Ag breakdown diagnostics. 


\section{Results and discussion}

\subsection{OES analysis}

The LPP spectra of Ag were recorded as a function of the emitted wavelength, distance from the target surface and delay time after the beginning of the laser pulse. Different authors ${ }^{17-30}$ have previously described this kind of study. In order to study the plasma-plume expansion dynamics of different species, we plotted spectrally resolved 1D-spatial and 1D-spectral imaging mode for different delay times.

Fig. 2 shows spatially and spectrally resolved optical emission images measured in the spectral range from about 290 to $360 \mathrm{~nm}$ following $1064 \mathrm{~nm}$ LPP of Ag in vacuum measured at three time delays of 0,20 and $100 \mathrm{~ns}$. These and all subsequent images show a linear false-color intensity displayed in the figures. Horizontal cuts through these images correspond to the wavelength-resolved spectra at a given distance from the target. In addition, from these images it is possible to obtain the atomic/ionic line shapes. The vertical axis in these images is the distance from the target surface. The emission features in this wavelength range and all the studied spectra are all attributable to $\mathrm{Ag}$ and $\mathrm{Ag}^{+}$. For the assignment of the spectra, we used the information tabulated in NIST Atomic Spectral Database. ${ }^{31}$ As we can see from these figures, at earlier times, especially below $80 \mathrm{~ns}$, the intense continuum radiation is dominant, mixed with $\mathrm{Ag}$ atomic and ionic lines. The continuum radiation for a LPP is due to result of Bremsstrahlung (free-free) and bound-free transitions. Bremsstrahlung is caused by collisions between free electrons and positively charged ions. ${ }^{2,32}$ The inverse process is called inverse Bremsstrahlung and is responsible for the absorption of laser radiation at or below the critical density region. The two intense lines at 328.068 and $338.289 \mathrm{~nm}$ of $\mathrm{Ag}$ (Fig. 2) are two resonance lines associated with the transitions $4 \mathrm{~d}^{10} 5 \mathrm{p}^{2} \mathrm{P}_{3 / 2}^{0} \rightarrow$ $4 \mathrm{~d}^{10} 5 \mathrm{~s}{ }^{2} \mathrm{~S}_{1 / 2}$ and $4 \mathrm{~d}^{10} 5 \mathrm{p}{ }^{2} \mathrm{P}^{0}{ }_{1 / 2} \rightarrow 4 \mathrm{~d}^{10} 5 \mathrm{~s}{ }^{2} \mathrm{~S}_{1 / 2}$, respectively. Beyond $100 \mathrm{~ns}$, the line-tocontinuum ratio increases and finally the spectrum consists of mainly atomic lines. 
(a)

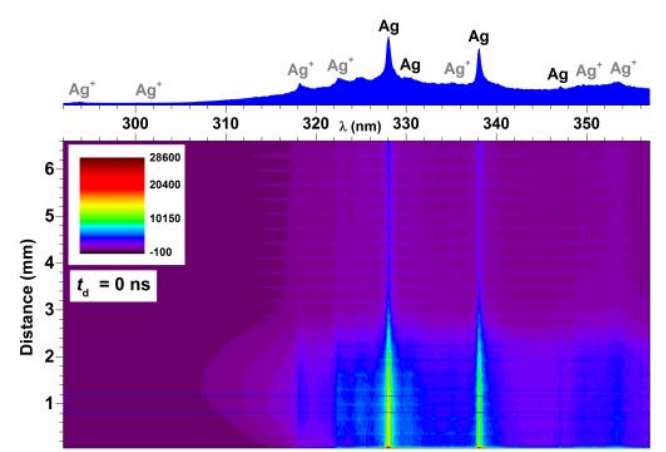

(b)

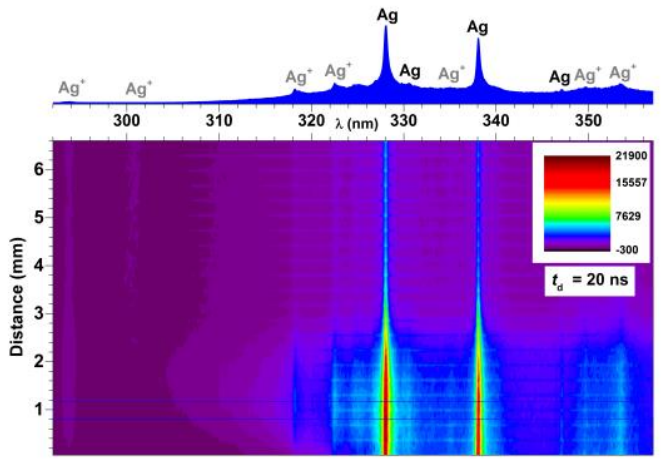

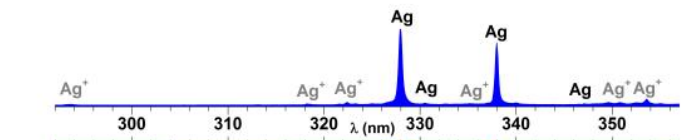

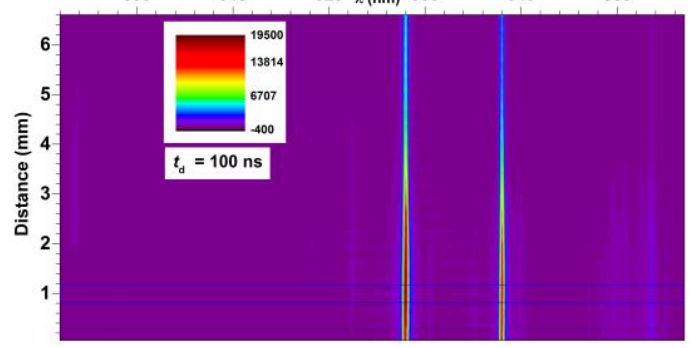

(c)

Fig. 2. Axial space-resolved image (bottom) of the Ag plasma $\left(5 \mathrm{~J} \times \mathrm{cm}^{-2}\right)$ at delays of 0 ns (a), $20 \mathrm{~ns}$ (b) and $100 \mathrm{~ns}$ (c), in all cases with a gate width of $100 \mathrm{~ns}$. The corresponding spectra (top) binned for the box indicated around $1 \mathrm{~mm}$ from the target surface are also shown.

Fig. 3 shows the spectra emitted from the Ag-LPP at different spatial distances $(z)$ from the target at 0 and $100 \mathrm{~ns}$. It is also shown in the inset of the figure $3 \mathrm{~b}$ a detailed assignment of the spectrum corresponding to a distance from the target of $1 \mathrm{~mm}$. At early times, especially below $80 \mathrm{~ns}$ (Fig. 3a at a delay of $0 \mathrm{~ns}$ ), continuum, ionic and atomic emission intensities in the plasma are greater in the region close to the $\mathrm{Ag}$ surface and decrease within a few millimeters away from the target. As time evolves > $80 \mathrm{~ns}$ (Fig. 3b at $100 \mathrm{~ns}$ ), the distribution of ionic and atomic lines moves away from the target surface. The spatial distributions for $\mathrm{Ag}$ and $\mathrm{Ag}^{+}$show different maxima, which varied with the delay time, but the behavior for the species with the same degree of ionization is very similar. 

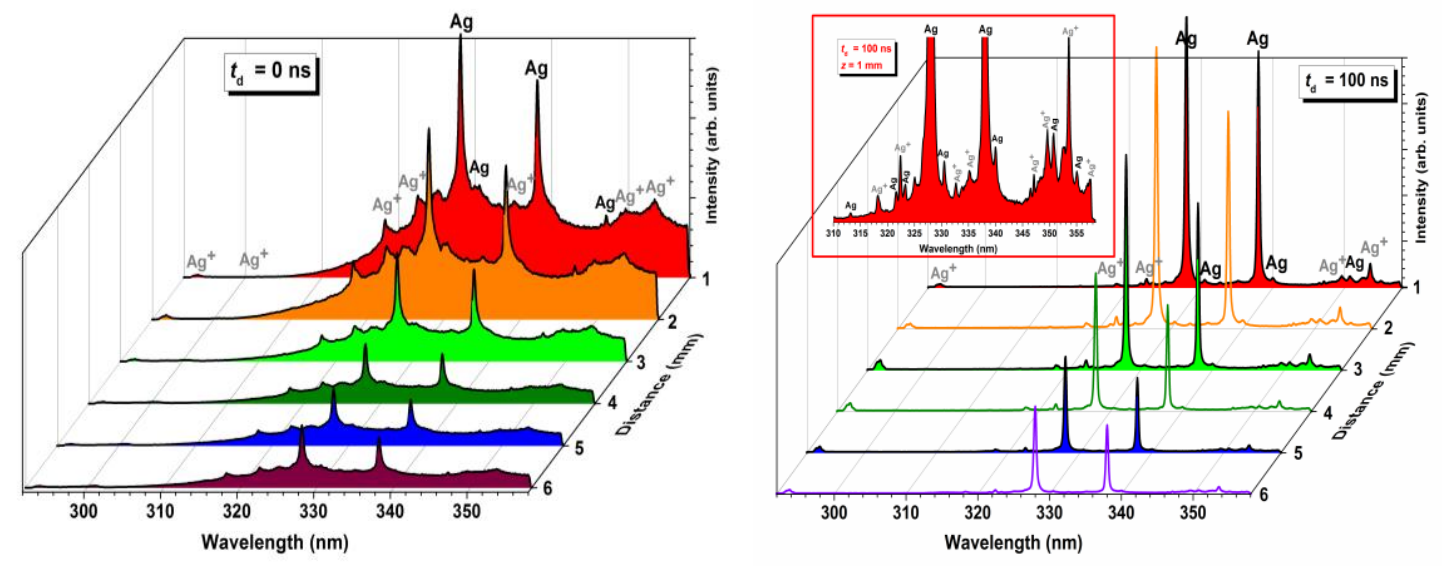

Fig. 3. The spatial distribution of the spectra emitted from Ag-LPP at delays of 0 ns (a), and $100 \mathrm{~ns}$ (b). The inset in Fig. 3b shows a detailed assignment of the spectrum at $z=1$ $\mathrm{mm}$.

Fig. 4 exhibits the time-resolved LPP spectra in the previous spectral region spatially integrated over $0 \leq z \leq 6.6 \mathrm{~mm}$. These spectra are dominated by the two previously mentioned $\mathrm{Ag}$ resonance lines and the continuum emission significantly contributes to the total emission for short delays $<80 \mathrm{~ns}$. As the delay increases, this emission drops significantly and spectral narrowing is observed as the consequence of the decrease of electron density and temperature. The temporal evolution of $\mathrm{Ag}$ and $\mathrm{Ag}^{+}$ line intensities has been used to construct the optical time-of-flight (OTOF) profiles shown in the inset of Fig. 4, obtained through integration of each of the emission lines, after baseline subtraction, for each delay time. The OTOF distributions of Ag (328.07 $\mathrm{nm}$, resonance line) and $\mathrm{Ag}^{+}(318.39 \mathrm{~nm})$ species were found to consist of one intense maximum at 60 and $40 \mathrm{~ns}$, respectively. The behavior of $\mathrm{Ag}(328.07 \mathrm{~nm}$, resonance line) is very similar to other $\mathrm{Ag}$ resonance lines $(338.29 \mathrm{~nm})$ but is slightly different to other non-resonance lines. Fig. 5 shows time-resolved spectra at different delay times in the spectral region from 380 to $440 \mathrm{~nm}$ for $0 \leq z \leq 6.6 \mathrm{~mm}$ and the normalized intensity dependence on delay for the species $\mathrm{Ag}(405.55 \mathrm{~nm})$ and $\mathrm{Ag}^{+}(408.59 \mathrm{~nm})$. The OTOF distributions of $\mathrm{Ag}\left(405.55 \mathrm{~nm}\right.$, non-resonance line) and $\mathrm{Ag}^{+}$(408.59 nm) species were 
found to consist of one intense maximum at 100 and $40 \mathrm{~ns}$, respectively. The behavior of $\mathrm{Ag}^{+}(408.59 \mathrm{~nm})$ is very similar to other $\mathrm{Ag}^{+}$lines (the line at $318.39 \mathrm{~nm}$ ) with a maximum at $40 \mathrm{~ns}$ and a lifetime of about $200 \mathrm{~ns}$. The emission intensity of $\mathrm{Ag}^{+}$species decreases faster than the emission intensity of Ag species. However, by comparing Ag resonance lines (the line at $328.07 \mathrm{~nm}$ ) and $\mathrm{Ag}$ non-resonance lines (the line at 405.55 $\mathrm{nm}$ ), the delay of the maximum is delayed for non-resonance lines. The resonance emissions, ending in the ground state are particularly susceptible to self-absorption and in more extreme cases can be self-reversed. ${ }^{33,34}$ Atomic species were detected up to about $2 \mu \mathrm{s}$.

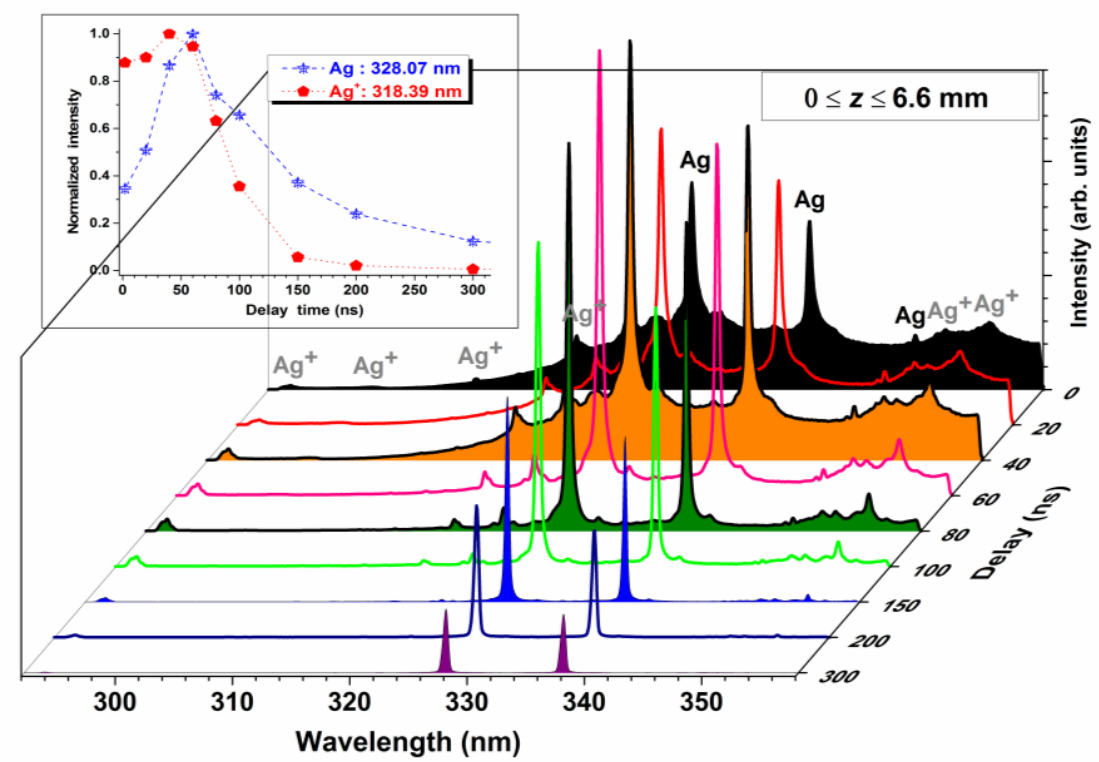

Fig. 4. Time-resolved Ag-LPP spectra at different delay times integrated over $0 \leq z \leq$ $6.6 \mathrm{~mm}$. The inset displays the normalized intensity dependence on delay time of the plasma ignition for the species $\mathrm{Ag}(328.07 \mathrm{~nm})$ and $\mathrm{Ag}^{+}(318.39 \mathrm{~nm})$. 


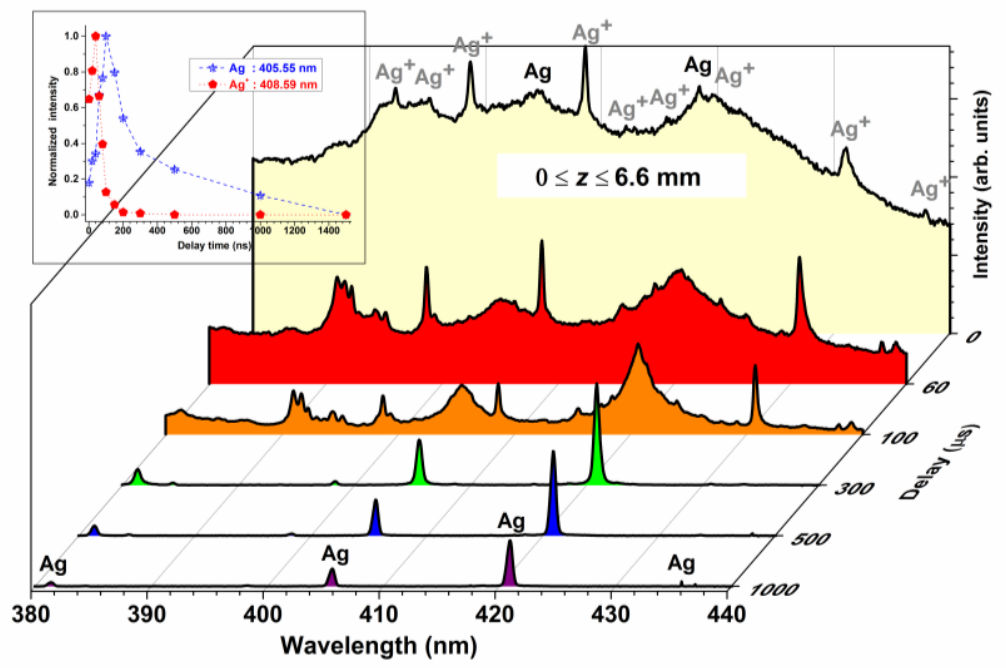

Fig. 5. Time-resolved Ag-LPP spectra at different delay times for $0 \leq z \leq 6.6 \mathrm{~mm}$. The inset shows the normalized intensity dependence on delay time for the species $\mathrm{Ag}$ $(405.55 \mathrm{~nm})$ and $\mathrm{Ag}^{+}(408.59 \mathrm{~nm})$.

\subsection{Velocity of plasma species}

The spatial and spectral images also allow detailed analysis of the $z$-dependence of each line intensity as a function of the delay. For studying time evolution velocities of resonance and non-resonance $\mathrm{Ag}$ atoms and $\mathrm{Ag}^{+}$species, we selected neutral $\mathrm{Ag}$ resonance emission at $328.07 \mathrm{~nm}\left(5 \mathrm{p}{ }^{2} \mathrm{P}_{3 / 2}^{0} \rightarrow 5 \mathrm{p}{ }^{2} \mathrm{~S}_{1 / 2}\right)$, Ag non-resonance line at $546.55 \mathrm{~nm}\left(5 \mathrm{~d}^{2} \mathrm{D}_{5 / 2} \rightarrow 5 \mathrm{p}^{2} \mathrm{P}^{0}{ }_{3 / 2}\right)$ and $\mathrm{Ag}^{+}$at $540.01 \mathrm{~nm}\left(4 \mathrm{~d}^{9}\left({ }^{2} \mathrm{D}_{5 / 2}\right) 4 \mathrm{f}^{2}[11 / 2]^{0}{ }_{6} \rightarrow 4 \mathrm{~d}^{9}\right.$ $\left.\left({ }^{2} D_{5 / 2}\right) 5 d{ }^{2}[9 / 2]_{5}\right) .{ }^{29}$ Fig. 6 shows the 1D-1D spectral images in the wavelength spectral range $495 \leq \lambda \leq 555 \mathrm{~nm}$ and vertical $\left(\mathrm{Ag}^{+}\right.$line profile versus distance from the target for the line at $540.01 \mathrm{~nm}$ ) and horizontally (spectrum for the box indicated around 1 $\mathrm{mm}$ ) contour profiles for time delays of 0 and $100 \mathrm{~ns}$. The emission features in this wavelength range are all attributable to $\mathrm{Ag}$ (non-resonance) and $\mathrm{Ag}^{+}$species. At earlier times $<60 \mathrm{~ns}$, the intense continuum and $\mathrm{Ag}^{+}$line at $540 \mathrm{~nm}$ dominate the spectrum while as time evolves ( $>80 \mathrm{~ns}$ ) Ag emission dominates and the continuum significantly decreases. 


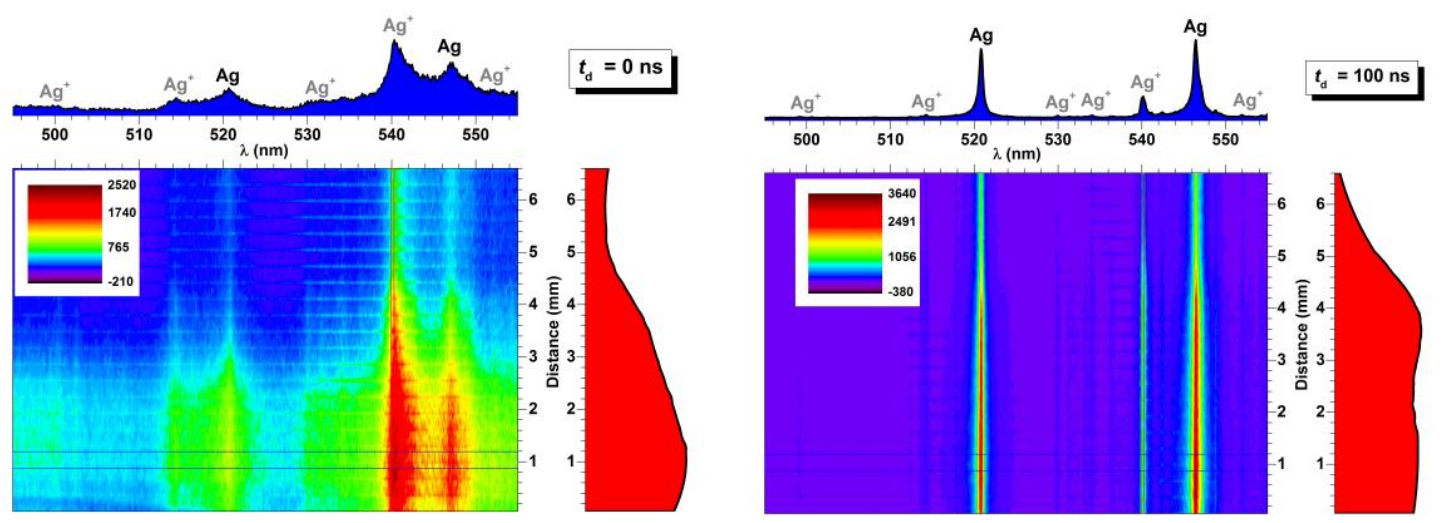

Fig. 6. Axial space-resolved image of the Ag plasma $\left(5 \mathrm{~J} \times \mathrm{cm}^{-2}\right)$ at delays of $0 \mathrm{~ns}$ (a), and $100 \mathrm{~ns}$ (b) and vertical (line profile for $\mathrm{Ag}^{+}$line at $540 \mathrm{~nm}$ ) and horizontal (spectrum around $1 \mathrm{~mm}$ ) contour profiles.

The changes of the emission distributions along the vertical line-profiles gives information on the propagation of the species from the target surface.

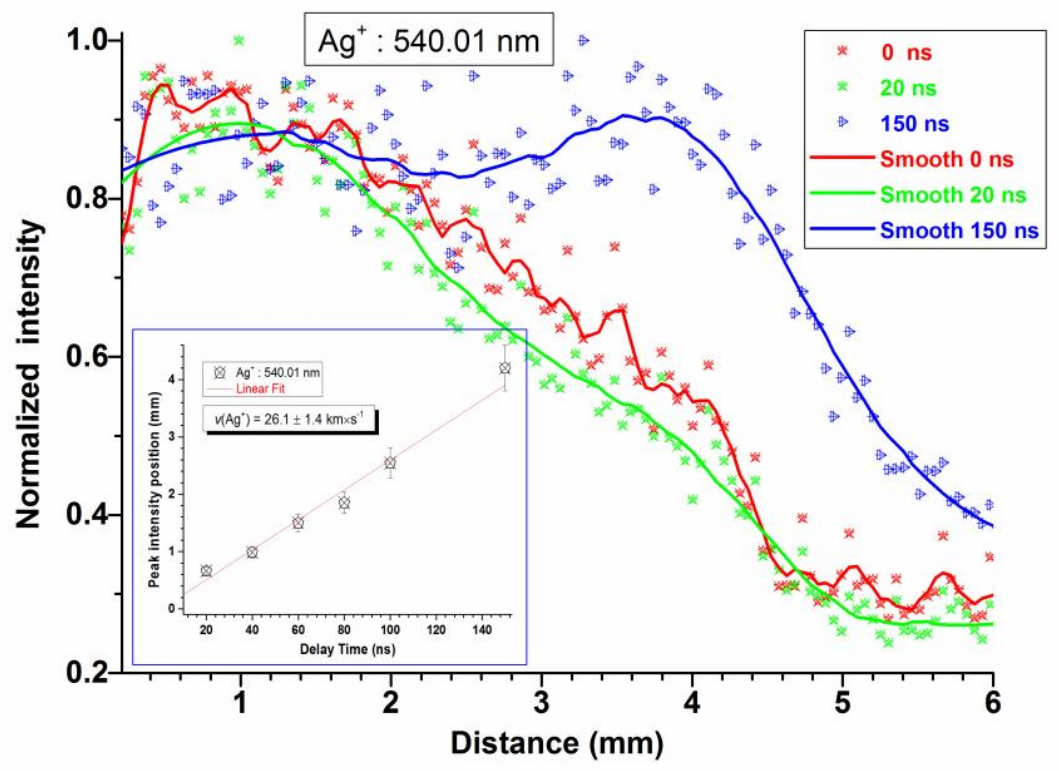

Fig. 7. Distance dependence intensities of the $\mathrm{Ag}^{+} 540.01 \mathrm{~nm}$ emission line measured for 0,20 and $150 \mathrm{~ns}$ following $1064 \mathrm{~nm}$ PLA of a silver target. Smoothed solid curves are also shown. The inset shows the variation of $\mathrm{Ag}^{+}$peak-intensity-position for different delays and a linear fit giving a velocity of $26.1 \pm 1.4 \mathrm{~km} / \mathrm{s}$. The error bars in the inset represent one standard deviation of uncertainty.

Fig. 7 shows the intensity of the $\mathrm{Ag}^{+} 540.01 \mathrm{~nm}$ emission as a function of the distance from the silver target, measured for delays of 0,20 and $150 \mathrm{~ns}$. The inset in this figure 
shows the variation of $\mathrm{Ag}^{+}$peak-intensity-position for different delays and a linear fit. The expansion velocity of $\mathrm{Ag}^{+}$ions is estimated from the position-time plot being, from the linear fit, $26.1 \pm 1.4 \mathrm{~km} / \mathrm{s}$, which corresponds to a kinetic energy of $380 \mathrm{eV}$. Fig. 8 displays the intensity of the Ag non-resonance $546.55 \mathrm{~nm}$ emission as a function of the distance from the target, obtained for delays of 100, 200, 300 and $400 \mathrm{~ns}$. It is interesting to note that the graphs obtained for 100 and 200 ns show a double maximum distribution. We believe that the maximum closest to the surface is possibly due to the neutral atoms directly removed from the surface and the other one can be associated to ion recombination. The inset in this figure shows the variation of Ag peak-intensityposition for different delays and the corresponding linear fit. The expansion velocity of Ag atoms estimated is $7 \pm 3 \mathrm{~km} / \mathrm{s}$, which corresponds to a kinetic energy of $27 \mathrm{eV}$.

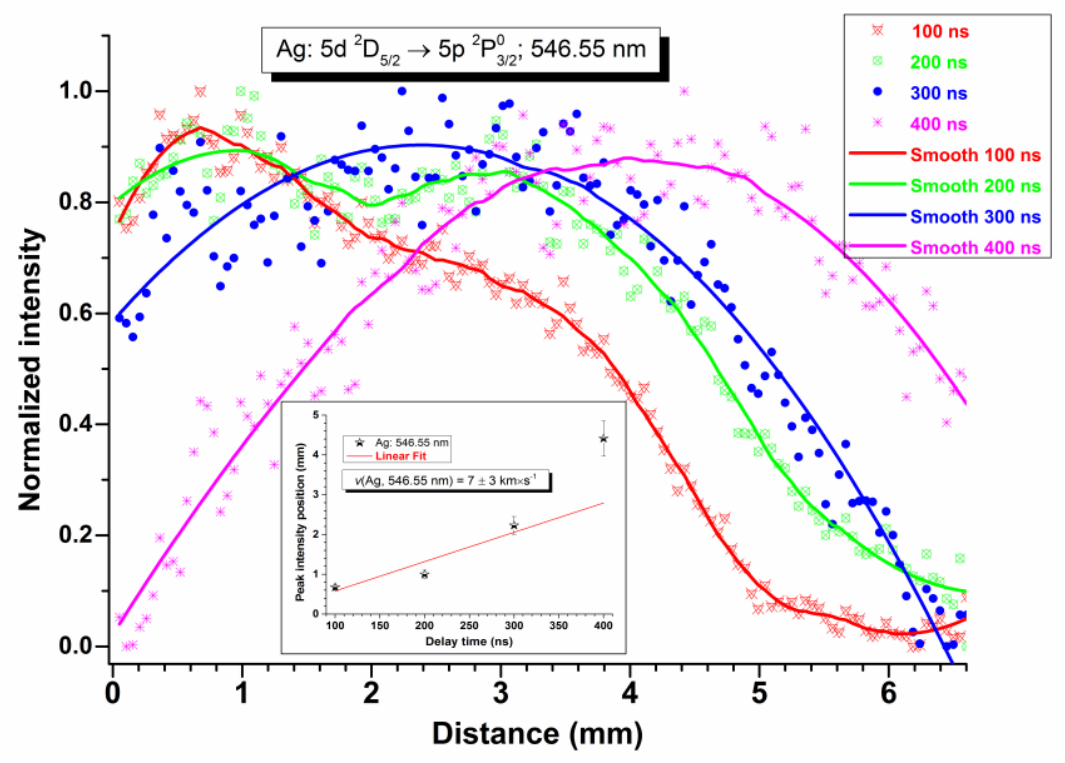

Fig. 8. Distance dependence intensities of the $\mathrm{Ag} 546.55 \mathrm{~nm}$ emission line measured for 100, 200, 300 and $400 \mathrm{~ns}$. Smoothed solid curves are also displayed. The inset shows the variation of $\mathrm{Ag}$ peak-intensity-position for different delays and a linear fit corresponding to a velocity of $7 \pm 3 \mathrm{~km} / \mathrm{s}$. The error bars in the inset plot represent one standard deviation of uncertainty.

The time-evolving spatial profile of the Ag $546.55 \mathrm{~nm}$ emission line measured at 20, 40, 60 and $80 \mathrm{~ns}$ is similar to the spatial profile at $100 \mathrm{~ns}$, showing that the effective velocity of atomic species for $<100 \mathrm{~ns}$ is virtually zero. The same behavior was also 
observed for the Ag resonance line at $328.07 \mathrm{~nm}$, but in this case, the estimated velocity was $16.3 \pm 1.3 \mathrm{~km} / \mathrm{s}$, notably greater than for non-resonance $\mathrm{Ag}$ lines. If we calculate the velocity using the Ag $546.55 \mathrm{~nm}$ line from $200 \mathrm{~ns}$, the estimated velocity is $15 \pm 2$ $\mathrm{km} / \mathrm{s}$, similar to the resonance line. There is an increase in the velocity of Ag species with distance from the target for times greater than $100 \mathrm{~ns}$ due to the initial acceleration of the species from zero velocity for $<100 \mathrm{~ns}$.

\subsection{Estimation of the plasma electron density}

The principal causes of spectral line broadening are the Doppler and Stark broadening, and spectrometer resolution. The Doppler broadening was estimated as $\Delta \lambda_{\text {FWHM }}^{\mathrm{D}}=7.16 \times 10^{-6} \times \lambda \times(T / M)^{1 / 2}$, where $\lambda$ is the line wavelength, $T$ the temperature in $\mathrm{K}$, and $M$ the atomic mass in amu. It was taken into account in the calculations although was very small $(\approx 0.01 \mathrm{~nm})$. In the present measurements $\Delta \lambda_{\text {instrument }}$ was $\approx 0.3 \mathrm{~nm}$. Stark line broadening from collisions with charged species is the primary mechanism influencing the LPP spectra. The FWHM of the Stark broadened line profile $\Delta \lambda^{\mathrm{S}}{ }_{\text {FWHM }}$ for neutral atoms or singly charged ions, after neglecting the ion contribution to the Stark-broadening, is connected to the electron density by the expression ${ }^{2,32}$

$$
\Delta \lambda_{\mathrm{FWHM}}^{\mathrm{S}} \approx 2 W\left(\frac{n_{\mathrm{e}}}{n_{\mathrm{r}}}\right)
$$

where $W$ is the Stark electron impact broadening parameter, $n_{\mathrm{e}}$ is the electron density in $\mathrm{cm}^{-3}$ and $n_{\mathrm{r}}$ is the reference electron density. The estimation of the electron density was done by fitting the emitted line profile to a Voigt function and by subtracting Doppler and instrumental line broadening, thus extracting the Lorentzian (Stark) component. The Ag lines at 328.07 and $338.29 \mathrm{~nm}$ have been used for this purpose. The Stark broadening parameter was taken from Dimitrijevic and Sahal-Brechot. ${ }^{35}$ By substituting the Stark line widths of these lines at different delay times in Eq. (1) and the electron impact parameter, $W(0.000465 \mathrm{~nm}(10000 \mathrm{~K}), 0.000523 \mathrm{~nm}(20000 \mathrm{~K})$, and 0.000589 
$\mathrm{nm}(30000 \mathrm{~K})$ for the line at $328.07 \mathrm{~nm}$ and $0.000443 \mathrm{~nm}(10000 \mathrm{~K}), 0.000500 \mathrm{~nm}$ $(20000 \mathrm{~K})$, and $0.000567 \mathrm{~nm}(30000 \mathrm{~K})$ for the line at $338.289 \mathrm{~nm}$ at a reference electron density $n_{\mathrm{r}}=10^{16} \mathrm{~cm}^{-3}$ ), we obtain the electron density. Although the temperature dependence of the Stark parameters is weak, ${ }^{32,35}$ it has been taken into account using appropriate interpolations. Fig. 9a gives the time evolution of electron density as a function of delay by spatially integrating in the region $0 \leq \mathrm{z} \leq 6.6 \mathrm{~mm}$, calculated from the two previously mentioned $\mathrm{Ag}$ lines.

(a)

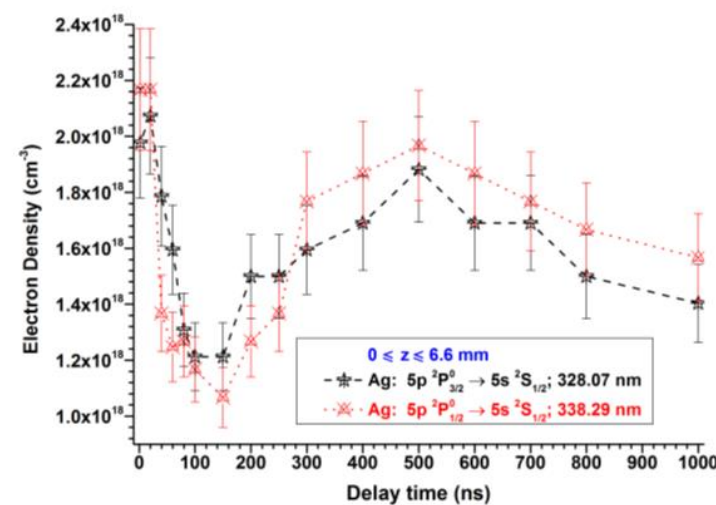

(b)

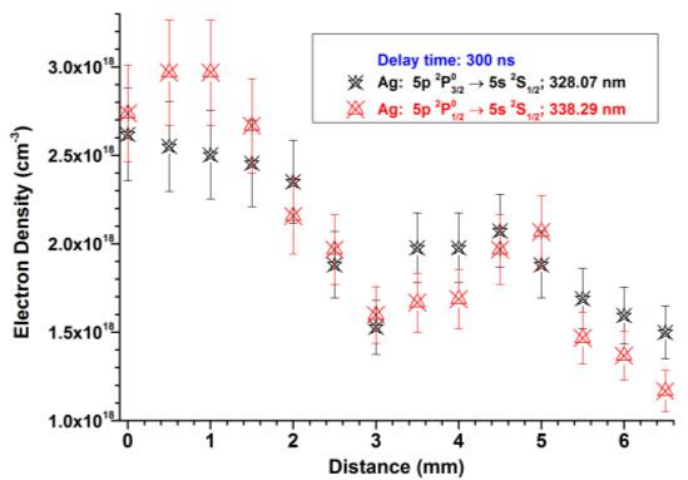

Fig. 9. Temporal $(0 \leq z \leq 6.6 \mathrm{~mm})$ (a) and spatial (300 ns) (b) evolution of electron density from Ag-LPP calculated from two Ag lines. The estimated error bars for electron density $(10 \%)$ result from the uncertainty in the impact parameters, temperature and line broadenings.

The initial electron density at $2 \mathrm{~ns}$ was approximately $2.1 \times 10^{18} \mathrm{~cm}^{-3}$. Afterwards, the density decreases until about $1.1 \times 10^{18} \mathrm{~cm}^{-3}$ over the period of $150 \mathrm{~ns}$ and then increases as the time is further increased up to $500 \mathrm{~ns}$. For $t_{\mathrm{d}}>500 \mathrm{~ns}, n_{\mathrm{e}}$ is slowly decreasing as the time is further increased. Since the emission line is partially masked by the continuum emission, the electron density for time $<100$ ns should be used with caution. The slow decay rate of electron density for time $>500 \mathrm{~ns}$ can be attributed to recombination. Fig. 9b shows the spatially resolved profile of the electron density along the axial direction at $300 \mathrm{~ns}$ delay from the laser pulse. In the observed spatial region, the electron number density roughly decreases with distance from the target. This 
behavior could be interpreted in terms of electron recombination processes in regions with high species number density. ${ }^{37}$

\subsection{Estimation of the electron temperature and ion stage distribution}

The plasma emission spectra have been simulated using the assumption of local thermodynamic equilibrium (LTE) and the system of Saha-Boltzmann equations based on the Saha equation

$$
\frac{N_{\mathrm{i}+1}}{N_{\mathrm{i}}}=\frac{2 Z_{\mathrm{i}+1}}{n_{\mathrm{e}} Z_{\mathrm{i}}}\left(\frac{2 \pi m_{\mathrm{e}} k T}{h^{2}}\right)^{3 / 2} \mathrm{e}^{-\epsilon_{\mathrm{i}} / k T},
$$

where $N_{\mathrm{i}}$ and $N_{\mathrm{i}+1}$ are the populations of the ionization stages $i$ and $i+1$, respectively, $Z_{\mathrm{i}}$ and $Z_{\mathrm{i}+1}$ are the partition functions of these states, $m_{\mathrm{e}}$ is the electron mass, $k$ is the Boltzmann constant, $h$ is the Planck constant, $n_{\mathrm{e}}$ is the electron density and $\varepsilon_{\mathrm{i}}$ is the ionization energy. The system of Saha equations (2) can be supplemented by the condition of electro-neutrality $\sum_{i=1} \mathrm{i} \cdot n_{\mathrm{i}}=n_{\mathrm{e}}$, where $n_{\mathrm{i}}$ is the density of species in the $i$-th state of ionization. In the simplest case of an optically thin and uniform plasma without self-absorption, the emission intensity of a radiative transition from an upper state $u$ to a lower state $l$ is given by the following expression, in terms of the spectral power per volume and solid angle: ${ }^{32}$

$$
I_{\mathrm{ul}}=\frac{1}{4 \pi} N_{\mathrm{u}} A_{\mathrm{ul}} \Delta E_{\mathrm{ul}},
$$

where $N_{\mathrm{u}}$ is the population of the upper state, $A_{\mathrm{ul}}$ the radiative spontaneous emission coefficient and $\Delta E_{\mathrm{ul}}$ the energy difference between the upper and the lower state.

For the simulation of the emissions, the Saha-Boltzmann NIST code available online ${ }^{31}$ was used. The spectral region 510-556 nm was selected, which contains $19 \mathrm{Ag}$ and $\mathrm{Ag}^{+}$ lines. As illustration, Fig. 10 shows a comparison of experimental spatially-integrated (0 $\leq z \leq 6.6 \mathrm{~mm}$ ) and simulated Saha-Boltzmann LTE spectra at 0 and $100 \mathrm{~ns}$ delays. The electron temperatures were adjusted until optimum agreement with experimental results 
was achieved. There is a good agreement between experimental and simulated spectra performed at the temperatures and densities indicated. The simulated Saha-Boltzmann LTE spectra included only the effects of Doppler broadening of the emission features at the estimated temperature. Our choice of $2 \times 10^{18} \mathrm{~cm}^{-3}$ as the electron density is taking into account our previous measurements.

(a)

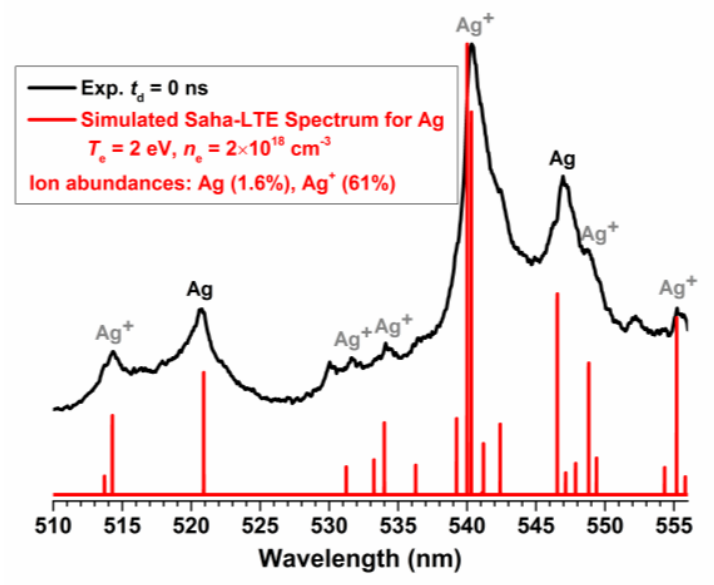

(b)

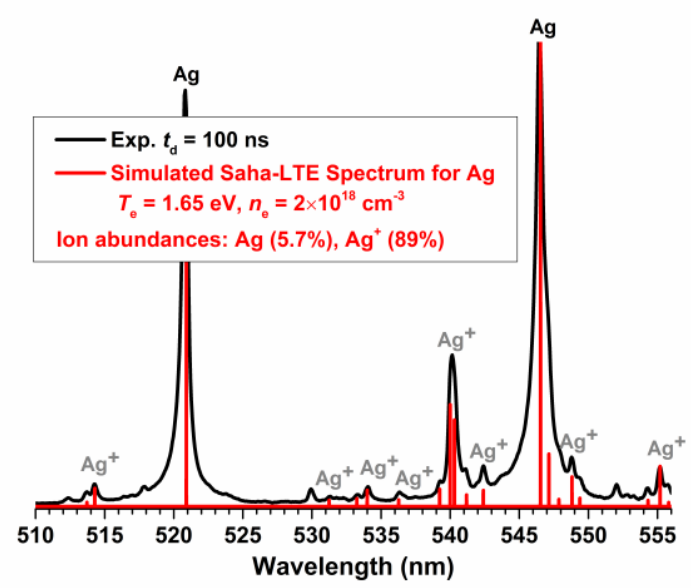

Fig. 10. Comparison of experimental spatially-integrated $(0 \leq z \leq 6.6 \mathrm{~mm})$ and simulated Saha-LTE spectra at (a) 0 ns delay at a temperature of $2 \mathrm{eV}$ and at an electron density of $2 \times 10^{18} \mathrm{~cm}^{-3}$ and (b) $100 \mathrm{~ns}$ delay at a temperature of $1.65 \mathrm{eV}$ and at an electron density of $2 \times 10^{18} \mathrm{~cm}^{-3}$.

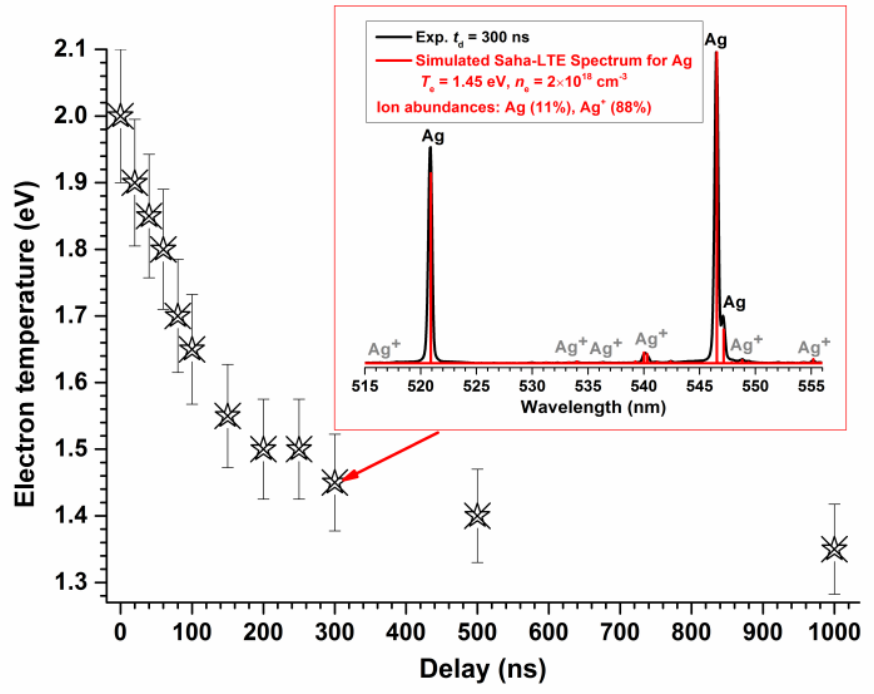

Fig. 11. Temporal variation in the temperature of the Ag spatially-integrated plasmaplume at $0-1000 \mathrm{~ns}$ delay time. Inset plot shows the experimental and simulated SahaLTE spectra in the $515-556 \mathrm{~nm}$ region at $300 \mathrm{~ns}$ delay. The estimated error bars for electron temperature $(10 \%)$ are due to the uncertainty in the intensity, transition probabilities and electron densities. 
Fig. 11 presents the time evolution of electron temperature for different delays from 0 to 1000 ns. The inset in Fig. 11 displays a comparison of experimental and simulated Saha-LTE spectra at $300 \mathrm{~ns}$ delay at a temperature of $1.45 \mathrm{eV}$ and at an electron density of $2 \times 10^{18} \mathrm{~cm}^{-3}$. The temperature is found to decrease in time from approximately $2 \mathrm{eV}$ at the first stages of plasma evolution to $1.3 \mathrm{eV}$ after $1 \mu \mathrm{s}$. The reduction of temperature with time is primarily due to three-body recombination between electrons and ions in the plasma. The values measured were well within the range of LPP temperatures and comparable to other related works published elsewhere. ${ }^{2,15,29,34}$ In addition, the simulated Saha-LTE spectrum provides relative ion concentrations of different plasma species. ${ }^{31}$ Fig. 12 displays the calculated atom/ion stage distribution in Ag LPP as a function of electron temperature at an electron density of $2 \times 10^{18} \mathrm{~cm}^{-3}$ as calculated with the Saha-LTE online utility. ${ }^{31}$ Silver atoms dominate at $T<1 \mathrm{eV}$ and $\mathrm{Ag}^{+}$at $1.1<T<$ $2.1 \mathrm{eV}$. As can be seen from Figs. 10a, 11 and 12, the most abundant ion at 0 ns $(T=2.0$ $\mathrm{eV}$ ) is $\mathrm{Ag}^{+}$having about $61 \%$ of the total population $\left(37 \% \mathrm{Ag}^{2+}\right.$ and $\left.1.6 \% \mathrm{Ag}\right)$. At 100 ns ( $T=1.65 \mathrm{eV}$, Figs. 10b, 11 and 12), the most abundant species is $\mathrm{Ag}^{+}$with $89 \%$ of the total population $\left(5.3 \% \mathrm{Ag}^{2+}\right.$ and $\left.5.7 \% \mathrm{Ag}\right)$. At $300 \mathrm{~ns}(T=1.45 \mathrm{eV}$, Figs. 11 and 12), the most abundant species is also $\mathrm{Ag}^{+}$with $88 \%$ of the total population $\left(1 \% \mathrm{Ag}^{2+}\right.$ and $11 \%$ $\mathrm{Ag})$. At $\mathrm{T}<0.6 \mathrm{eV}$, the only possible species is $\mathrm{Ag}$.

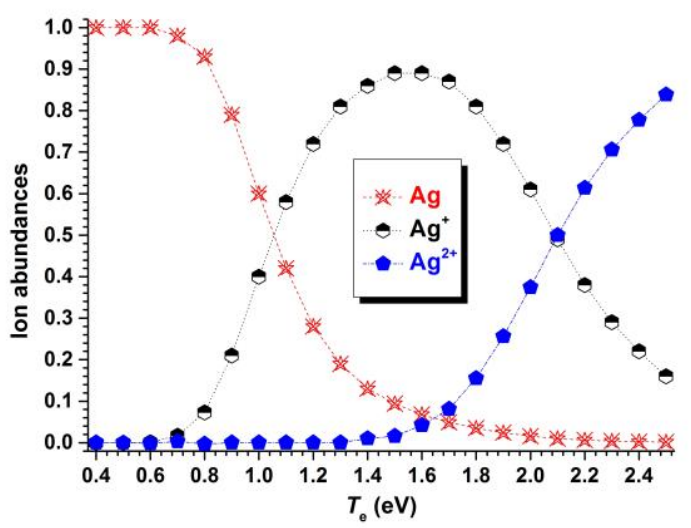

Fig. 12. Calculated ion stage distribution in a steady-state Ag plasma as a function of electron temperature at an electron density of $2 \times 10^{18} \mathrm{~cm}^{-3}$. 
There are two main recombination processes in plasmas, three-body (collisional) and photo-recombination (radiative). The difference is based on what particle removes excessive energy and momentum when an electron and an ion recombine, being another electron in three-body recombination (TBR) and a photon in photo-recombination (PHR). The recombination rate coefficient for these processes is related to the probability of transition from the free electron state (continuum) into a bound state of the resulting atom. The rate of recombination can be calculated by considering the relaxation times of TBR and PHR processes given by $^{38}$

$$
\begin{aligned}
& \tau_{\mathrm{TBR}} \approx 1.1 \times 10^{26} Z^{3} T^{9 / 2} n_{\mathrm{e}}{ }^{-2}, \\
& \tau_{\mathrm{PHR}} \approx 3.7 \times 10^{12} Z^{2} T^{3 / 4} n_{\mathrm{e}}^{-1},
\end{aligned}
$$

where $Z$ is the ionic charge, $T$ the electron temperature in $\mathrm{eV}$ and $n_{\mathrm{e}}$ the electron density in $\mathrm{cm}^{-3}$. For the LPP studied here, $n_{\mathrm{e}}$ is in the range $(1.1-3.0) \times 10^{18} \mathrm{~cm}^{-3}$ and $\mathrm{T} \approx 1.3-$ $2.0 \mathrm{eV}$, yielding values for the relaxation time of the TBR process of $0.1-0.2 \mathrm{~ns}$ and $1.7-2.4 \mu$ s for the relaxation time of the PHR process. From these values we see that $\tau_{\mathrm{TBR}}$ is even less than the duration of the laser pulse and therefore the TBR process significantly influences the plasma dynamics during the laser pulse, while the effect of PHR can be considered negligible.

In the calculation of electron or plasma temperature, we have considered the plasma to be in LTE. The condition that atomic/ionic states should be depopulated or populated mostly by electron collisions, rather than by radiation, requires an electron density high enough to ensure a high collision rate. In other words, the number of collisions, which is linked to the electron density, must be sufficiently large to ensure LTE is satisfied. According to the McWhirter criterion, ${ }^{39,40}$ the electron density $n_{\mathrm{e}}{ }^{\text {crit }}\left(\mathrm{cm}^{-3}\right)$ should pass a threshold

$$
n_{\mathrm{e}}^{\text {crit }} \geq 1.6 \times 10^{12} \sqrt{T}(\Delta E)^{3},
$$


where $T$ in $\mathrm{K}$ is the plasma temperature and $\Delta E$ in $\mathrm{eV}$ is the largest energy transition for which the condition holds. As we mentioned before, in the estimation the electron temperature, we use several lines of $\mathrm{Ag}$ and $\mathrm{Ag}^{+}$in the spectral region 510-556 nm. At the evaluated temperatures in the range of 15000 to $23000 \mathrm{~K}$ (Fig. 11), for the $\mathrm{Ag}^{+}$ transition with the largest energy-gap $\Delta \mathrm{E}=2.38 \mathrm{eV}$, equation (6) yields $(2.6-3.3) \times$ $10^{15} \mathrm{~cm}^{-3}$. The values of $n_{\mathrm{e}}$ estimated from the Stark-broadened lines is approximately three orders of magnitude greater than the values calculated from Eq. (6), implying that the condition necessary for the LTE approximation is valid.

\section{Conclusions}

The laser-induced plasma of silver in vacuum produced by a nanosecond highpower Nd: YAG laser at $1064 \mathrm{~nm}$ has been studied spatially and temporally by OES. The emissions generated are principally caused by electronic relaxation of excited neutral $\mathrm{Ag}$ atoms and ionized $\mathrm{Ag}^{+}$. The significant efforts of this work have been focused on the spatial and temporal study of 1D-1D OES data. Average expansion velocity, electron density and temperature measurements have been performed from the analysis of 1D-1D spectral images. The velocity measurements have been carried out by tracking the maximum intensity position versus the distance from the target at different delays. A slightly different behavior has been observed between Ag resonant and nonresonant lines. Ag electronically excited atoms move with low velocities compared to electronically excited $\mathrm{Ag}^{+}$ions. The effective velocity of $\mathrm{Ag}$ atoms for resonance and non-resonance lines at earlier times, especially bellow $100 \mathrm{~ns}$, is practically zero. The spatial and temporal behavior of the electron densities have been reported by using the Stark broadening method. The electron temperature measurements have been performed by a comparison between Ag atomic/ionic Saha-LTE spectra and the experimental 
spectra at various delay times from the laser pulse incidence. Electron densities in the range $(1-3) \times 10^{18} \mathrm{~cm}^{-3}$ were estimated.

\section{Conflicts of interest}

The authors declare no conflicts of interest.

\section{Acknowledgments}

This research was supported by the Spanish Ministry of Economy and Competitiveness (MINECO) through Project CTQ2016-75880-P.

\section{References}

1. D. B. Chrisey and G. K. Hubler (Eds.), Pulsed Laser Deposition of Thin Films, Wiley, New York, 1994.

2. L. J. Radziemski and D. A. Cremers (Eds.) Laser-Induced Plasmas and Applications, Dekker, New York, 1989.

3. M. Oujja, M. Sanz, F. Agua, J. F. Conde, M. García-Heras, A. Dávila, P. Oñate, J. Sanguino, J. R. Vázquez de Aldana, P. Moreno, M. A. Villegas and M. Castillejo, J. Anal. At. Spectrom. 2015, 30, 1590-1599.

4. J. P. Sylvestre, S. Poulin, A. V. Kabashin, E. Sacher, M. Meunier and J. H. T. Luong, J. Phys. Chem. B, 2004, 108, 16864-16869.

5. C. L. Haynes, P. Richard and R. P. V Duyne, J. Phys. Chem. B, 2001, 105, 55995611.

6. C. Phipps, M. Birkan, W. Bohn, et al., J. Propuls. Power, 2010, 26, 609-637.

7. P. K. Kennedy, D. X. Hammer and B. A. Rockwell, Prog. Quantum Electron., 1997, 21, 155-248.

8. S. Moncayo, A. Marín-Roldán, S. Manzoor, J.J. Camacho, V. Motto-Ros, J.O. Caceres, J. Anal. At. Spectrom. 2018, 33, 1884-1891.

9. R. S. Harmon, F. C. De Lucia, A. W. Miziolek, K. L. McNesby, R. A. Walters, P. D. French, Geochemistry: Exploration, Environment, Analysis, 2005, 5, 21-28.

10. M. López-Arias, M. Oujja, M. Sanz, R. A. Ganeev, G. S. Bolataev, N. K. Satlikov, R. I. Tugushev, T. Usmanov and M. Castillejo, J. Appl. Phys., 2012, 111, 043111. 
11. M. Oujja, J.G. Izquierdo, L. Bañares, R. de Nalda, M. Castillejo, Phys. Chem. Chem. Phys., 2018, 20, 16956-16965.

12. R. C. Issac, K. V. Pillai, S. S. Harilal, et al. Appl. Surf. Sci., 1998, 125, 227-235.

13. D. Margarone, L. Torrisi, A. Borrielli and F. Caridi, Plasma Sources Sci. Technol., 2008, 17, 035019.

14. O. K. Shuaibov, M. P. Chuchman and L. L. Shimon, Ukr. J. Phys., 2004, 49, 866869.

15. M. Musadiq, N. Amin, Y. Jamil, M. Iqbal, M. A. Naeem and H. A. Shahzad, Int. J. Engineering Technol., 2013, 2, 32-43.

16. M. Esposito, T. Lippert, C. W. Schneider, et al., J. Optoelectronics Adv. Mat., 2010, 12, 677-680.

17. J. Siegel, G. Epurescu, A. Perea, F. J. Gordillo-Vazquez, J. Gonzalo and C. N. Afonso, Optics Lett., 29, 2228-2230.

18. D. Doria, K. D. Kavanagh, J. T. Costello and H. Luna, Meas. Sci. Technol., 2006, 17, 1-5.

19. H. Luna, J. Dardis, D. Doria and J. T. Costello, Braz. J. Phys., 2007, 37, 13011305.

20. A. De Giacomo, M. Dell'Aglio, D. Bruno, R. Gaudiuso and O. De Pascale, Spectrochim. Acta Part B, 2008, 63, 805-816.

21. M. Chen, X. Liu, M. Zhao, C. Chen and B. Man, Phys. Rev., 2009, 80, 016405.

22. A. De Giacomo, M. Dell'Aglio, R. Gaudiuso, G. Cristoforetti, V. Palleschi and E. Tognoni, Spectrochim. Acta Part B, 2008, 63, 980-987.

23. D. Campos, S. S. Harilal and A. Hassanein, Appl. Phys. Lett., 2010, 96, 151501.

24. R. W. Coons, S. S. Harilal, M. Polek and A. Hassanein, Anal. Bioanal. Chem., 2011, 400, 3239-3246.

25. S. S. Harilal, T. Sizyuk, A. Hassanein, D. Campos and P. Hough, J. Appl. Phys., 2011, 109, 063306.

26. T. Wu, X. Wang, S. Wang, J. Tang, P. Lu and H. Lu, J. Appl. Phys., 2012, 111, 063304.

27. L. Diaz, J. J. Camacho, J. P. Cid, M. Martin and J. M. L. Poyato, Appl. Phys. A, 2014, 117, 125-129.

28. J. J. Camacho, L. Diaz, S. Martinez-Ramirez and J. O. Caceres, Spectrochim. Acta Part B, 2015, 111, 92-101.

29. H. Liu, B.S. Truscott and M. N. R. Ashfold, Plasma Sources Sci. Technol., 2016, 25, 015006. 
30. H. Liu, B. S. Truscott and M. N. R. Ashfold, Scientific Reports, 2016, 6, 25609.

31. NIST atomic spectra database online at http://physics.nist.gov/PhysRefData/ ASD/index.html/ 2018.

32. H. R. Griem, Principles of Plasma Spectroscopy, Cambridge University Press, Cambridge, 1997.

33. V. Lazic, R. Barbini, F. Colao, R. Fantoni and A. Palucci, Spectrochim. Acta Part $B, 2001, \mathbf{5 6}, 807-820$.

34. A. M. E. Sherbini, A. E. E. Sherbini and C.G. Parigger, Atoms, 2018, 6, 44.

35. M. S. Dimitrijevic and S. Sahal-Brechot, J. Research Physics, 1999, 28, 291-294.

36. S. S. Harilal, T. Sizyuk, A. Hassanein, D. Campos and P. Hough, J. Appl. Phys., 2011, 109, 063306.

37. A. De Giacomo, M. Dell'Aglio, R. Gaudiuso, G. Cristoforetti, V. Palleschi and E. Tognoni, Spectrochim. Acta Part B, 2008, 63, 805-816.

38. J. F. Ready, Effects of High-Power Laser Radiation, Academic, New York, 1971.

39. R. W. P. McWhirter, in: R.H. Huddlestone, S.L. Leonard (Eds.), Plasma Diagnostic Techniques, Academic Press, New York, 1965, pp. 201-264, Chapter 5.

40. G. Cristoforetti, A. De Giacomo, M. Dell'Aglio, S. Legnaioli, E. Tognoni, V. Palleschi and N. Omenetto, Spectrochim. Acta Part B, 2010, 65, 86-95. 